\title{
La virtualidad en la tutoría docente: Una aproximación a su análisis desde la universidad española
}

\author{
Virtuality in Tutoring: An Approach to Analysis of Spanish Universities \\ A virtualidade na tutoria docente: uma abordagem a sua análise desde a universidade espanhola
}

Gustavo Toledo Lara* (http://orcid.org/0000-0002-5104-9555)

Facultad de Humanidades y Ciencias Sociales, Universidad Isabel I, Burgos, España
Recibido: 25-11-16 Revisado: 23-05-17 Aceptado: $14-06-17$

Publicado:18-12-17
RESUMEN. El proceso reformista universitario europeo requiere una nueva visión de la relación entre el docente y el estudiante. En este artículo se analiza cómo el contexto universitario español ha demandado adaptaciones en el rol del docente en relación con las nuevas tecnologías identificando la relevancia de la tutoría virtual como oportunidad para comprender otro sentido del proceso de enseñanza y aprendizaje. Desde lo cualitativo se aborda el tema de estudio para caracterizar el contexto universitario español y la tutoría virtual. Se identifica la necesidad de formación didáctica al docente, más allá del fomento a la investigación académica además de proponer una tipología de la tutoría virtual a partir de cinco niveles. Finalmente, Bolonia centra su atención en el estudiante, sin embargo se tiende a continuar con las mismas prácticas anteriores a la reforma. La tutoría virtual junto al factor motivacional se ubica como una de las acciones más determinantes.
Palabras clave: enseñanza superior, aprendizaje en línea, tutoría, personal académico docente, reforma de la educación.

ABSTRACT. The European university reform process requires a new approach into the relationship between faculty and students. This paper analyzes how the Spanish university context has demanded adaptations for the faculty's role in the presence of new technologies, identifying the relevance of virtual tutoring as an opportunity to understand another meaning of the teaching and learning process. From a qualitative standpoint,

Key words: higher education, online learning, tutoring, the topic of study is addressed in order to characterize the Spanish university context and

Citar como: Toledo Lara, G. (2017). La virtualidad en la tutoría docente: una aproximación a su análisis desde la universidad española.

Revista Digital de Investigación en Docencia Universitaria, 11(2), 323-342. doi: http://dx.doi.org/10.19083/ridu.11.509

*E-mail: gustavotoledolarađgmail.com 
virtual tutoring. The faculty's training need is identified beyond promoting academic research, in addition to proposing a new classification of virtual tutoring based on five levels. Finally, Bologna centers its attention on the student; however, the practices established before the reform are still ongoing. Virtual tutoring, together with the

RESUMO. O processo da reforma universitária europeia exige uma nova visão da relação entre professor e aluno. Este artigo analisa como o contexto universitário espanhol exigiu adaptações no papel do professor frente às novas tecnologias, identificando a relevância da tutoria virtual como uma oportunidade de compreender outra forma do processo de ensino e aprendizagem. A partir do enfoque qualitativo, o tema de estudo é abordado para caracterizar o contexto universitário espanhol e a tutoria virtual. Identifica-se a necessidade de formação didática do professor além da promoção da pesquisa académica, e propõe-se uma tipologia de tutoria virtual a partir de cinco níveis. Finalmente, Bologna centra a atenção no aluno; no entanto, tende a continuar com as mesmas práticas anteriores à reforma. A tutoria virtual com o fator motivacional classifica como uma das ações mais decisivas.

En los últimos veinte años la universidad española ha experimentado un notable crecimiento, de manera particular la universidad pública. Además de esto, estamos dentro de una realidad social altamente volátil la cual experimenta cambios en forma constante con respecto a los ámbitos que tradicionalmente se consideraban que eran medianamente estables o que dicha realidad no se modificaba con una velocidad tal y como la observamos actualmente (Quintero, 2014). Por su parte, las investigaciones de Fernández Enguita (1999) y Angoitia y Rahona (2007) coinciden en el hecho de considerar el carácter masificador del sistema universitario español. A juicio de Angoitia y Rahona (2007):

El crecimiento del sistema universitario español ha jugado un papel fundamental, principalmente desde comienzos de la década de los noventa. Así, mientras que la expansión de la enseñanza obligatoria fue el fenómeno predominante durante las décadas de los setenta y ochenta, en los últimos años la intensidad del cambio ha sido mayor en el ámbito universitario (p. 246).

Ahora bien, el Sistema Universitario Español (SUE) se presenta como un conjunto conformado por dos tipos de universidades según su nivel de adscripción, en el que se establece una diferenciación entre las universidades públicas y las privadas. Sin embargo, también existen las denominadas universidades a distancia y las universidades especiales. Respecto de este punto, Roselló, Olivares y Pujolras (2009) señalan que:

El importante crecimiento del número de universidades en España arranca de la Ley de Reforma Universitaria de 1983 que promovió el proceso de modernización y descentralización de la 
educación universitaria, y la colaboración y transferencia de competencias en esta materia con las comunidades autónomas (p. 8).

En un intento de construir una tipología de las instituciones que conforman el SUE, se pueden caracterizar las instituciones de educación universitaria de la siguiente manera (ver Tabla 1).

Tabla 1

Tipo y descripción de las universidades en España

Tipo

Universidad

pública

niversidad

privada

niversidad

a distancia

Universidad

especial

\section{Descripción}

Las universidades públicas son aquellas instituciones creadas por Ley de la Asamblea Legislativa de la Comunidad Autónoma en cuyo ámbito territorial vayan a establecerse y también aquellas creadas por Ley de las Cortes Generales, a propuesta del Gobierno y de acuerdo con la Comunidad Autónoma donde se ubiquen.

Son universidades privadas aquellas creadas por personas físicas o jurídicas en virtud del apartado 6 del artículo 27 de la Constitución Española, y con sometimiento a lo dispuesto en la Ley Orgánica 6/2001 de Universidades.

Son universidades que ofrecen estudios universitarios bajo el modelo del e-learning y que pueden ser tanto públicas como privadas. La oferta educativa contempla los tres ciclos formativos igual que las universidades presenciales.

Son universidades especiales aquellas que ofrecen enseñanza de segundo y tercer ciclo, especializándose así, en la oferta de cursos de posgrado lespecialización, máster y doctorado). Pueden ser instituciones públicas o privadas.

Además de las instituciones anteriores existe en España un conjunto de establecimientos conocidos como instituciones no universitarias, diferentes a las universidades tradicionales que ofrecen formación académica orientada hacia el dominio de una técnica profesional específica por medio de cursos, especializaciones o másteres (Dávila, 2008). A este tipo de diferenciación institucional dentro de la educación superior se le conoce como sistema binario. Por otro lado, también está el sistema integrado, el cual se desarrolló mediante reformas dentro de las mismas universidades con la creación de carreras cortas, generalmente vinculadas con las carreras largas, y dado el proceso de armonización con el Espacio Europeo de Educación Superior (EEES), este tipo de carreras cortas está en vías de extinción. Sin embargo, esta diversificación de los sistemas de educación superior ha conformado parte del debate que sobre dicho sistema se hace. A juicio de Dávila (2008):

Desde las posiciones críticas se argumenta que la diversificación constituye una forma de reproducción de la desigualdad social, debido a que el origen socioeconómico de la matrícula no universitaria es generalmente más bajo que el de la universitaria y el título tiene menor reconocimiento social y por lo tanto salarial. Otros autores observan beneficios en la medida que la diferenciación es una respuesta tendiente a la equidad porque logra abrir las puertas de la 
educación superior a una mayor cantidad de estudiantes y, de no existir, se excluiría a gran parte de los sectores menos favorecidos (...) Desde otro punto de vista, la diversificación institucional puede entenderse como una oferta de mayo-res opciones educativas para los alumnos, así como también de mayores oportunidades laborales para profesores e investigadores (p. 4).

La complejidad de la naturaleza del SUE sugiere un punto de revisión que es de imperativa necesidad. Se trata del rol del profesor universitario en todo este proceso que ocurre en las universidades. Este punto es multifactorial en su revisión y análisis ya que se identifica, por ejemplo, la influencia que ha recibido el profesor durante su experiencia como estudiante, la motivación y vocación docente, el desequilibrio entre la relevancia de la investigación sobre la docencia, los planes de formación de las universidades dirigidos a los profesores y el acercamiento del profesor al uso de las tecnologías de la información y la comunicación.

Desde luego, al concebirse un nuevo estilo educativo, la práctica diaria y la cotidianidad institucional van mermando las iniciativas innovadoras logrando con ello que en muchos casos las universidades sean víctimas del peso de su propia tradición, por lo tanto, los cambios paradigmáticos dentro del mundo universitario suelen ser sumamente lentos. A propósito de este punto, Kindelán (2013) afirma que:

En efecto, las políticas universitarias y los órganos de ejecución de las mismas se han propuesto la implementación de planes estratégicos (vid. “Estrategia Universidad 2015 para la modernización de la universidad española") que otorguen mayor flexibilidad en la organización de las “enseñanzas”, una diversificación curricular, unas metodologías docentes centradas en el estudiante y una perspectiva del aprendizaje que se extiende a lo largo de toda la vida. Pero la realidad es que la práctica va muy por detrás de la teoría, el peso de la tradición hace que la enseñanza en nuestras aulas siga impartiéndose de la misma forma -un modelo centrado en el profesor y en los conocimientos- y la nueva estructura organizacional de la misma, basada en el aprendizaje, está aún en un proceso de instauración incipiente (p. 29).

Dadas las consideraciones anteriores, se persigue con este artículo ofrecer criterios y líneas de discusión en torno al papel del profesor universitario centrando la atención en el rol que este juega como tutor, guía o acompañante del estudiante. En este sentido, la acción del profesor universitario dentro del contexto de la enseñanza virtual ofrece la posibilidad de la revisión de tal dinámica a propósito de la ya palpable presencia e impacto de las nuevas tecnologías dentro del proceso tanto de enseñanza como de aprendizaje, sin dejar de reconocer que, en la práctica de la tutoría virtual se transmiten y comparten conocimientos pero también valores y actitudes (Martín Izard, 2014) por lo tanto, es una idea más que reafirmada que el proceso de Bolonia centra su atención e interés en el estudiante y es allí en donde el profesor acompaña, asesora y orienta al futuro profesional. Sin embargo, hay que reconocer que si antes de Bolonia, la acción docente se inclinaba mayoritariamente a la clase magistral y con pocas iniciativas de contacto con el estudiante, durante el desarrollo del proceso de Bolonia ese mismo profesor sigue inclinándose a continuar con las mismas prácticas.

Es necesario reafirmar que, aunque el centro de atención recae sobre el estudiante universitario, el profesor definitivamente va a generar el contexto propicio para facilitar procesos tanto grupales como individuales, con lo cual, la acción tutorial es aquella tarea que los docentes de este sector educativo 
ejercen con sus estudiantes y desde luego es un aspecto fundamental al momento de identificar la intención de personalizar la enseñanza. (Martín Izard, 2014). Al hablar de personalizar la enseñanza no se trata de diseñar un currículo a la carta, se trata más bien de una actividad didáctica diseñada desde el reconocimiento de los intereses y posibilidades de cada estudiante y cómo esta realidad puede orientarse con relación a su desarrollo integral y desde luego profesional.

Ahora bien, desde un punto de vista más práctico y menos normativo, el carácter virtual de la educación superior impone la necesidad de una redefinición del rol del profesor universitario. Esté convencido o no, la misma dinámica de lo virtual obliga a repensar el tipo de vinculación que el docente tiene con el estudiante. Este hecho ubica la acción tutorial más personalizada en la que la motivación, acompañamiento y cercanía de parte del docente cobra una importancia significativa que incide enormemente en la prosecución de los estudios por parte de los alumnos.

Además de lo anterior, es justo reconocer que, dentro del proceso reformista impulsado por el EEES, una de las grandes ausencias precisamente es la formación y preparación del profesorado universitario respecto de la didáctica requerida por un nuevo modelo educativo (Toledo Lara, 2015). Se destaca mayoritariamente el logro investigativo y en menor proporción, la docencia y la preparación didáctica. En este orden de ideas, resulta familiar encontrar profesores con una dilatada vida investigativa y con innumerables publicaciones, pero con poca o escasa preparación didáctica, lo cual no se debe comprender solo como la competencia para revisar el currículo sino que debe ir más allá y capacitar a los profesores universitarios en cuanto a rol de orientadores y facilitadores de un proceso que el mismo estudiante debe diseñar y concebir y lógicamente, ese profesor debe tener las herramientas necesarias para aprovechar su formación académica en beneficio de sus estudiantes. Todo ello determinado en este contexto, desde la virtualidad en la tutoría docente.

Este artículo se compone de dos bloques relacionados entre sí, pero diferenciados en cuanto a su reconocimiento: el primer bloque ofrece un acercamiento al proceso de Bolonia y la integración universitaria española con el objeto de contextualizar la discusión que aquí se persigue acompañado de las aportaciones de diferentes autores que estudian sobre este aspecto. En el segundo bloque se desarrollará con mayor profundidad el rol del profesor universitario como tutor de la educación virtual. En este punto se pretende ofrecer: (a) una visión global de lo que se entiende como tutoría, (b) los retos a los que se enfrenta el mundo docente a esta nueva concepción de su acción, y (c) una tipología de la tutoría virtual como producto del proceso crítico, reflexivo e investigativo desarrollado hasta el presente. Por último y como balance final, se presentan las conclusiones a las que se desemboca como fruto de esta discusión.

\section{CUERPO METODOLÓGICO DEL TRABAJO}

La identificación de la perspectiva teórico metodológica reviste una lógica procedimental que procura la conjugación de varias facetas que, a modo experiencial, logra sumergir al investigador dentro del tema objeto de estudio. En este sentido, los procesos cognitivos y el bagaje teórico permiten comparar, contrastar, comprender e inferir las diversas realidades que se desprenden de la observación del fenómeno estudiado. En definitiva, “todo conocimiento tiene, como punto de partida, el momento 
en que surge una pregunta que genera inquietud y, por consiguiente, es el detonante para indagar y descubrir" (Toledo Lara, 2017, p. 8).

Dada la naturaleza de la presente investigación se empleó el análisis de las fuentes documentales primarias y secundarias. Esta técnica busca el estudio de un caso o fenómeno por medio de documentos elaborados por el hombre social. Tal técnica fue fundamental para este trabajo, ya que brindó aportes significativos para el análisis al que se pretendió llegar. Por otro lado, este acercamiento a las fuentes permitió conocer el estado del arte sobre el objeto de estudio de la investigación: aproximación a las investigaciones previas, estado del arte del tema objeto de estudio, enfoques teóricos y metodológicos, conclusiones, entre otros.

Por otra parte, al analizar los documentos respectivos este tipo de perspectiva metodológica precisa de igual modo alguna herramienta o programa informático diseñado para el análisis de contenido cualitativo. No obstante, el diseño de esta investigación es compatible con la oportunidad de descubrir, explorar, inferir, establecer relaciones causales y desde luego, la función interpretativa. Por lo tanto, y sin dejar de reconocer las herramientas tecnológicas para análisis cualitativo, se consideró con la debida densidad teórica, asumir la construcción de patrones o líneas de lectura con la finalidad de afinar y precisar los procesos y las interacciones a los que hubiere lugar, al momento del acercamiento e investigación de las fuentes.

Las fases establecidas para el desarrollo del presente estudio fueron las siguientes: (1) Planteamiento del problema, preguntas de investigación y objetivos, (2) Revisión de la literatura y formulación de proposiciones, (3) Obtención y transcripción de los datos, (4) Análisis global, (5) Análisis profundo, y (6) Balance final a modo de conclusión.

Respecto de la dimensión correspondiente de la reflexión que aquí se presenta, es importante destacar la visión cualitativa y hasta holística como característica sustancial, sin dejar de reconocer que, en el contexto educativo, "la investigación educativa debe ser una actividad científica y por tanto formal, sistemática, controlada, empírica y objetiva” (Tejedor, 2014, p. 177). Por lo tanto, el método cualitativo al tener una serie de criterios que le caracterizan, se concibe como un proceso dinámico dentro de la investigación propiamente dicha, "de tal manera, que, dentro de la investigación cualitativa, es parte relevante la garantía del grado de validez como producto de la rigurosidad con la que se desarrolla la investigación" (Toledo Lara, 2017, p. 19). En este orden de ideas, se señalan a continuación los aspectos sustantivos a este estudio y su descripción (ver Tabla 2).

\section{EL PROCESO DE BOLONIA Y LA INTEGRACIÓN UNIVERSITARIA ESPAÑOLA}

Uno de los hechos que ha determinado la dinámica universitaria española fue el inicio de la implantación a partir del 2003 del Plan de Bolonia. En ese año se presenta el documento La integración del sistema universitario español en el Espacio Europeo de Enseñanza Superior por parte del Ministerio de Educación, Cultura y Deporte. Para tal fin fue necesaria la homologación de los estándares que rigen la vida universitaria para adaptarlos a los lineamientos del referido plan. Así, y según Santoveña (2010) “La Declaración de Bolonia busca alcanzar una Europa del conocimiento como elemento imprescindible 
Tabla 2

Aspectos sustantivos de la investigación y su descripción

Dimensión

Nivel de la

investigación

Perspectiva
Epistemológica

\section{Descripción}

\begin{abstract}
Nivel analítico-interpretativo: este nivel indica que el investigador observa, analiza e interpreta el objeto de estudio, es decir, se produce la hermenéutica en tanto que los datos o las partes de un todo se presten a diferentes interpretaciones. (Martínez Carrazo, 2006, p. 135)
\end{abstract}

\begin{abstract}
Epistemología construccionista: desde esta perspectiva, se asume que diferentes personas puedan construir diversos significados en relación con un mismo fenómeno. Parte del construccionismo social, cuyos principales exponentes son: Berger y Lukman, Hegel y Marx. Dirige su atención hacia la construcción social del conocimiento. (Sandín, 2003, p. 49)
\end{abstract}
Perspectiva
Teórico -
Epistemológica
Perspectiva interpretativa: se pretende la comprensión del significado de los fenómenos sociales. Esta postura desarrolla interpretaciones de la vida social y el mundo desde una perspectiva cultural e histórica. (Sandín, 2003, p. 56)
Perspectiva
Teórico -
Epistemológica

para el desarrollo social y la formación de los ciudadanos" (p. 1). No obstante, la puesta en marcha de esta línea de acción se lleva a cabo en España cinco años después de su sanción. Este hecho colocó a España en una posición diferente con relación a un grupo de países europeos que ya contaban para entonces con un camino recorrido respecto de la implantación del referido plan. De tal modo, RuizGallardo y Castaño (2008) apuntan que:

La Universidad española se encuentra en una situación excesivamente alejada de la realidad social y económica en la que vivimos. El enfoque del EEES puede subsanar estas deficiencias, pero la adaptación conlleva, en mayor o menor grado, una transformación del modelo educativo actual. (p.10).

En este orden de ideas, el paso de una perspectiva educativa a otra conlleva dentro de sí una serie de retos a los que las mismas instituciones universitarias debieron y deben hacer frente. La metamorfosis de una concepción centrada en las horas lectivas del profesor a una concepción centrada en las labores de aprendizaje por parte del estudiante supone desde luego un conjunto de acciones que aspiren al menos en teoría, a ir modificando nuestra posición ante el hecho educativo universitario, con lo cual, la manera en la que comprendemos la universidad como concepto y como institución siempre va a estar "bajo los influjos de corrientes filosóficas que determinan el deber ser, en este caso, de la educación universitaria" (Toledo Lara, 2015, p. 4). A propósito de este punto Sanjurjo (2012) apunta que: La implantación del Espacio Europeo de Educación Superior (EEES), proceso que se inició con la Declaración de La Sorbona (Sorbonne Joint Declaration, 1998) y que alcanzó su punto de 
inflexión con la Declaración de Bolonia de 19 de junio de 1999, supuso en España la superación de los créditos LRU, para cuyo cálculo se computaban únicamente las horas lectivas teóricas y prácticas, y la aparición en escena de los créditos ECTS o European Credit Transfer System, que además de aquellas contabiliza las horas que los alumnos deberán emplear para el estudio, la preparación y la realización de exámenes. (p. 122).

Más allá ly no menos importante) de la administración del currículo universitario, se trata de la adaptación de la institución universitaria a los nuevos criterios orientadores de su función acordados a nivel europeo y entendidos de manera particular por parte de las autoridades españolas. En este sentido, Llinàs-Audet, Girotto y Salé (2011) señalan la necesidad de la adopción por parte de las universidades de un proceso de revisión interna, es decir:

Las universidades que no adopten nuevas estrategias o no traten de, por lo menos, considerar los numerosos cambios que se propagan en torno a la educación superior, se enfrentarán a los probables dilemas que incluyen una mayor debilidad de carácter político y fiscal, el desgaste en la moral de los profesores y personal administrativo y la capacidad de atracción del talento (p. 38).

Ante esta nueva concepción educativa, y frente a los requerimientos que esta exige, se hace necesaria la formación didáctica dirigida al profesorado universitario, sin embargo, tal y como se ha señalado inicialmente, este aspecto ha sido una de las ausencias más palpables dentro de este proceso reformista, aunque siendo justos, se pueden reconocer algunas iniciativas en este particular, pero aún dichas iniciativas no han adquirido el carácter de políticas de Estado. A propósito de este punto, es revisable la afirmación de Ruiz-Gallardo y Castaño (2008), al asegurar que:

El papel del profesor universitario debe cambiar. Esto nos va a resultar difícil y costoso. La mayoría de los profesores universitarios somos especialistas en nuestra área de conocimiento y no nos hemos formado en aspectos didácticos ajenos a la misma. Con el paso de los años, nos hemos acostumbrado a impartir nuestras clases en la forma tradicional, tal y como nos enseñaron a nosotros. Además, las estructuras universitarias están adaptadas a este sistema de Clases Magistrales. (p. 9).

Vinculada a la idea anterior, se puede avanzar un poco más allá con respecto al análisis que aquí se pretende, revisando la postura de Del Pozo Andrés (2008), con relación a la aplicación del proceso reformista de Bolonia, quien señala que:

La aplicación del plan de Bolonia en muchas universidades europeas, en sus aspectos metodológicos, no ha resultado tan novedosa ni tan traumática como puede ser en España por la simple razón de que ya se habían implantado desde hace años sistemas basados en el trabajo personal del alumno. Por eso en la mayoría de los países no se ha debatido tanto como en el nuestro sobre este aspecto, pero tampoco se han llevado a cabo planes sistemáticos de formación del profesorado ni de información sobre las cuestiones más relevantes del Espacio Europeo de Educación Superior. (p. 64)

Ante estas aseveraciones, es casi obligada la revisión de lo que establece el Proceso de Bolonia como criterios orientadores para los países signatarios del mismo ya que la critica que ha estado presente en el debate tanto político como académico, no siempre se ha acompañado de la revisión y 
análisis a profundidad de lo que se entiende a nivel europeo respecto de los grandes cambios que han de suscitarse a partir de la adscripción a la reforma universitaria, con lo cual se hace oportuno comprender la dimensión y sentido de la crítica, a la par de un proceso de observación sobre lo que realmente se ha establecido como línea de acción, y lo que en efecto se ha llevado a la práctica. Es decir, es necesaria la revisión a profundidad de los documentos aprobados a nivel europeo para el sector universitario.

Al revisar los documentos oficiales expuestos a nivel europeo, se pueden identificar una serie de aspectos que conforman el corpus ideológico y operativo que acompaña la puesta en marcha del mencionado proceso reformista. A continuación, se presenta una tabla contentiva de cuatro áreas consideradas por Bolonia acompañadas de sus respectivos criterios (ver Tabla 3). Dichas áreas han sido establecidas una vez se han identificado los campos de correspondencia de las líneas de acción acordadas para la educación superior europea.

Una vez revisada la tabla anterior, podemos evocar la máxima que acompaña el Proceso de Bolonia respecto a que "ahora el estudiante es el centro de atención". Sin embargo, todas las acciones conducentes para lograr este objetivo mayoritariamente se han centrado tanto en la nueva administración curricular como en la administración y gobierno universitario. Si bien es cierto que se ratifica la autonomía académica y universitaria de las instituciones respectivas, no hay mención expresa de una política o línea de acción que asegure la atención o formación al profesorado universitario, no así con el caso de la sinergia entre educación y otros sectores (investigación, empresas). Sin embargo, se señala el impulso de métodos innovadores de enseñanza, pero los mismos se dejan a disposición de las universidades para que las mismas puedan crear sus planes internos de formación dirigidos a los profesores y dentro de ellos el tema de las nuevas tecnologías como uno de los aspectos más relevantes de las corrientes didácticas contemporáneas.

En el caso español las universidades están dentro de la Comunidad Autonómica que les pertenece obedeciendo a su ubicación geográfica. De tal modo, y producto del proceso de descentralización y traspaso de funciones, las Comunidades Autónomas establecen sus propios criterios de asignación de fondos a partir del Producto Interno Bruto (PIB) que se produce en las regiones, el número de estudiantes y el número de profesores entre otros factores, lo que desemboca en una desigual proporción del gasto dirigido hacia las universidades, es por eso que no hay una distribución con patrones comunes. Este contexto junto con la reducción de la financiación experimentada en los últimos años ha provocado que la investigación desarrollada sea reorientada en sus fines y modos, es decir, se provocó una nueva manera de financiación universitaria a partir de la investigación que en ella se produce.

Dentro de este particular, las universidades tendieron a impulsar la investigación desde alianzas estratégicas con sectores privados de la economía. Esto dependió principalmente del corte institucional de cada una de ellas, es decir, mientras el perfil de la Universidad era compatible con las ciencias o la empresa, la transferencia del conocimiento producido por las investigaciones se inclinarían hacia estos campos y por lo tanto tendrían la oportunidad de contar con más ingresos por este concepto, mientras que aquellas universidades de corte más social y humanístico, trataron de impulsar aquellas Facultades dedicadas a la ciencia con el objeto de hacerlas más competitivas y atractivas a la inversión privada. Mientras esto ocurría, la docencia trataba de abrirse paso ante las contracciones económicas 


\section{Tabla 3}

Criterios orientadores según el Proceso de Bolonia discriminados por áreas de acción

Área

Criterio orientador según el Proceso de Bolonia

\begin{tabular}{|c|c|}
\hline Área & Criterio orientador según el Proceso de Bolonia \\
\hline Estudiantil & $\begin{array}{l}\text { Educación superior centrada en el estudiante. } \\
\text { Movilidad estudiantil. } \\
\text { Aprendizaje a lo largo de la vida. } \\
\text { Mejora de la empleabilidad de los titulados. } \\
\text { Reducción de la exclusión universitaria por razones económicas. }\end{array}$ \\
\hline $\begin{array}{l}\text { Administración } \\
\text { y gobierno } \\
\text { universitario }\end{array}$ & $\begin{array}{l}\text { Políticas públicas en función del proceso de Bolonia. } \\
\text { Integración de la formación e investigación. } \\
\text { Promoción de la cooperación europea. } \\
\text { Apoyo en decisiones financieras favorables para el proceso EEES. } \\
\text { Sinergia entre educación y otros sectores (investigación, empresas). } \\
\text { Garantizar que las universidades cuenten con recursos necesarios. } \\
\text { Financiación pública como prioridad y desarrollo sostenible de las universidades. } \\
\text { Apertura de diálogo sobre financiación y gobernanza de la Educación Superior. } \\
\text { Planes nacionales para mejorar la calidad. } \\
\text { Dimensión social de la educación universitaria. } \\
\text { Reconocimiento de la autonomía académica y universitaria. } \\
\text { Desarrollo de gestión eficiente en las estructuras de las instituciones universitarias. } \\
\text { La Universidad como servicio público. } \\
\text { Promoción internacional del sistema universitario. }\end{array}$ \\
\hline $\begin{array}{l}\text { Currículo } \\
\text { universitario }\end{array}$ & $\begin{array}{l}\text { Impulso del ECTS (conocido como el crédito europeo) } \\
\text { Promoción del aprendizaje de idiomas } \\
\text { Máster de corta duración y Doctorado más extenso. } \\
\text { Cooperación europea en el aseguramiento de la calidad. } \\
\text { Estudios de 1er ciclo de } 3 \text { años mínimo. } \\
\text { Adopción de un sistema de titulaciones reconocible y comparable. } \\
\text { Red de trabajo en el nivel doctoral. } \\
\text { Formación interdisciplinar en los estudios doctorales. } \\
\text { Reforzar la reglamentación sobre el tercer ciclo. } \\
\text { EEES estructurado en } 3 \text { ciclos. } \\
\text { Programas conjuntos y planes de estudio flexibles. } \\
\text { Facilitar el aprendizaje sobre el medio ambiente. } \\
\text { Impulsar métodos innovadores de enseñanza. } \\
\text { Reconocimiento del aprendizaje no formal e informal. }\end{array}$ \\
\hline $\begin{array}{l}\text { Personal } \\
\text { docente }\end{array}$ & Movilidad docente. \\
\hline
\end{tabular}


y aunque existiesen iniciativas y motivaciones plausibles de no pocos profesores, la práctica docente era eclipsada por el destello de la investigación y con ella la acción tutorial tendía a debilitarse o a circunscribirse al rol de preguntas y respuestas sin involucrar acciones motivadoras o integradoras que asegurasen el menor impacto del paso por parte del estudiantado de la educación presencial a la educación virtual.

\section{LA TUTORÍA VIRTUAL: ALGUNAS CONSIDERACIONES}

A nivel global se puede asegurar que existe consenso en cuanto a reconocer la relevancia del rol del docente universitario con respecto a la tutoría que ha de desarrollarse dentro de la universidad, ya que, “si realmente apostamos por un proceso de innovación educativa, debemos considerar al docente como eje fundamental en dicho proceso" (Hernández, 2014, p. 33). A partir del enfoque educativo centrado en el estudiante, la tutoría cobra singular importancia por cuanto se pretende que, al menos en teoría, se estimule la autogestión del aprendizaje con la compañía y apoyo del profesor. Más específicamente dentro del campo de la educación virtual este rol juega un papel determinante ya que la imagen de la universidad ante el estudiante es precisamente el profesor.

En este sentido, lograr la empatía y asegurar la continuidad en los estudios definitivamente tiene que ver con el apoyo que el estudiante pueda recibir de parte del docente que le acompaña. Así y de acuerdo con Martínez, Pérez y Martínez Juárez (2014):

En estos tiempos de cambio y reformulaciones en la Educación Superior, uno de los tópicos en el escenario de la innovación educativa es la tutoría universitaria. Su protagonismo reside tanto en el importante papel que desempeña en relación con la formación integral de los estudiantes, como en el rol que juega como uno de los ejes articuladores del enfoque educativo centrado en el alumnado y el aprendizaje. (p. 270).

Sin embargo, es justo reconocer que si en la educación universitaria presencial, el ejercicio de la tutoría se ve influenciado por la motivación y disposición del profesor universitario para con sus estudiantes, en la educación virtual esa misma tutoría debe contar no solo con dichas características sino que además, se debe lograr componer una fórmula en la que se asegure el acompañamiento, el dominio de las e-actividades y la presencia frecuente del profesor en los espacios virtuales para el intercambio y la comunicación, pasando así a ser un gestor del conocimiento que fomenta el aprender a aprender (Hernández \& Martín, 2011). En definitiva, la tutoría virtual cuenta con un fuerte componente vivencial, es decir, se desarrolla a partir de las experiencias y convicciones propias del profesor tal y como Giner, Muriel y Toledano (2013) señalan:

Los profesores universitarios llegan al ejercicio de la profesión sin formación en materias pedagógicas, psicológicas y sociológicas y esta formación la adquieren en la práctica docente y a partir de sus propias experiencias. Igualmente, cada profesor desempeña su labor tutorial según sus experiencias, motivación por enseñar, expectativas, confianza, optimismo, preocupaciones sobre la enseñanza, su percepción del papel y responsabilidad como tutor. (p. 90)

Ahora bien, la valoración que se tiene de la investigación sobre la docencia hace que efectivamente se crea que el profesor universitario se va formando en la práctica en cuanto a los 
aspectos fundamentales del ejercicio docente propiamente dicho. Por lo tanto, el cambio hacia un modelo centrado en el estudiante no se puede generar de manera automática, menos aun si no se establecen estrategias institucionales conducentes a la formación didáctica, con lo cual se plantean una serie de incertidumbres y desafíos que no pueden ni deben pasar desapercibidos, en aquellos programas de formación que las universidades estén concibiendo para su profesorado (Hernández \& Martín, 2011). Esta situación hace que se siga reproduciendo el modelo centrado en la enseñanza, aunque se crea que se está avanzando en el modelo centrado en el aprendizaje sin dejar de reconocer que, en la mayoría de los casos, existe un deficiente impacto de la docencia en el progreso profesional. (López, Pérez-García \& Rodríguez, 2015).

En este orden de ideas, la tutoría a distancia se ubica como un punto de interés complejo. Los procesos y características tanto cognitivas como procedimentales que caracterizan esta acción conducen obligatoriamente a reconocer más allá de su relevancia, el reconocimiento de que efectivamente el profesor universitario acompaña y orienta la experiencia del futuro profesional que se está formando, por lo tanto, parece obvio reconocer el esfuerzo necesario por formar al profesorado universitario para que pueda dar respuesta a los nuevos retos que se le presentan (Hernández, 2014). Una manera de comprender la dimensión de la tutoría a distancia la ofrece Dopico Rodríguez (2013), al proponer las características de esta acción desde el escenario educativo (ver Tabla 4).

\section{Tabla 4}

Características de la tutoría a distancia

\section{La tutoría a distancia}

El/la Tutor/a realiza un seguimiento
personalizado del proceso de
aprendizaje, ofrece los recursos
didácticos disponibles y trata de
cubrir a distancia los ámbitos
propios de la Tutoría, siendo el
referente Orientador que facilita las
herramientas de estudio, indicando
cómo manejar los materiales y los
recursos didácticos disponibles.

El/la Tutor/a ayuda a comprender, a extraer lo esencial, a analizar y relacionar los contenidos, a resolver dudas y a explicar puntos concretos de especial dificultad en los contenidos de las materias que se cursan.

\author{
En función de su papel evaluador, \\ convierte la evaluación en un \\ elemento más de la tutoría a \\ través de comentarios escritos \\ que incluyen consejos y \\ orientaciones para mejorar la \\ progresión de los aprendizajes.
}

Tal y como se desprende de las características anteriores, se evidencia que más allá del dominio de una disciplina o campo de estudio, el factor motivacional y empático es de singular importancia al momento de emprender una tutoría virtual. Sin embargo, no se puede aventurar sin tener una formación adecuada para que precisamente la acción docente no se vea eclipsada exclusivamente por los logros investigativos. En este orden de ideas, en una investigación realizada por Borges (2005) se identificó que dentro de los motivos por los cuales los estudiantes de educación virtual se frustran, es el no tener cercanía de parte del docente, no recibir respuesta o responder muy tarde, o tener una 
presencia esporádica o nula en el aula virtual, es decir, según el mismo autor:

Aun siendo el estudiante un elemento central, el docente debe ser consciente de las acciones o carencias en las que él mismo puede incurrir. A pesar de sus funciones facilitadoras y de guía, con el acierto o la torpeza de sus acciones los docentes pueden influir de manera determinante en la motivación o en la frustración de sus estudiantes. Así mismo, dentro de la educación virtual la motivación personal juega un papel determinante. El paso de una educación presencial a una virtual genera un impacto en el estudiante que lo hace más vulnerable en cuanto a sus expectativas e intereses personales. Por lo tanto, la tutoría virtual sin presencia activa y efectiva sencillamente es inviable en este caso ya que "la función tutorial es uno de los pilares sobre los que se consolida la educación a distancia" (Rodríguez, 2014, p. 53). Si bien es cierto, que los espacios virtuales de aprendizaje aportan una serie de retos de cara a la intencionalidad formativa, al hablar de tutoría, “tenemos que insistir en que la herramienta no realiza la función tutorial, esta solo la realiza un tutor, una persona con intencionalidad educativa, que es capaz de compartir los intereses del aprendiz y de dotar de significado estos aprendizajes" (Martín Izard, 2014, p. 119)

Otro aspecto para observar es la concepción que se tiene sobre la educación virtual, ya que al ser un adulto que se está formando, se entiende de manera equivocada que él mismo por sí solo puede ser capaz de afrontar todos los requerimientos propios de este tipo de aprendizaje. Además de ello, el sentimiento de soledad y de poco interés hacia él como estudiante, puede inducir al abandono de los estudios. Es más, mientras que en los países más industrializados el abandono de los estudios obedece mayoritariamente a nuevas oportunidades laborales, en el caso español hay un fuerte componente motivacional en los estudiantes que desertan. (Cabrera, 2015).

Una vez en este punto, es importante reconocer lo que se acepta como acción tutorial en virtud del contexto universitario que aquí se trata. De acuerdo con Rodríguez (2014) estamos “entendiendo la acción tutorial como el conjunto de tareas de quien interactúa con el educando a distancia, respecto de los contenidos y procesos de aprendizaje" (p. 51). En este sentido, se puede proponer una tipología de la tutoría virtual con el objeto de identificar sus características sustanciales y desde luego profundizar tanto en su estudio como en la revisión de su puesta en marcha dentro del entorno virtual del proceso de aprendizaje. Para la elaboración de la siguiente tabla, se consideraron las características más representativas del docente dentro del entorno de formación virtual y el despliegue de su acción en función del proceso de aprendizaje por parte del estudiante (ver Tabla 5).

Tal y como se desgrana de la clasificación anterior, la acción del docente se ubica en una posición relevante dentro del proceso de tutoría en el entorno virtual, ya que el profesorado universitario constituye el elemento clave en cualquier reforma dirigida a este sector educativo (Hernández \& Martín, 2011). Esta observación requiere de manera imperativa una formación didáctica aplicada que pueda servir de soporte a dicha experiencia. Sin embargo, el conocimiento que se tenga ante las herramientas informáticas no debe suponer el dar por sentado que se domina su uso ya que por sí mismas no constituyen el aseguramiento de una formación determinada, es decir, las herramientas y recursos para la formación en línea deben ser empleadas para tal fin una vez que se conciban las líneas de acción y se logre la didáctica aplicada idónea para estos casos. Por lo tanto, “la acción tutorial 
llevada a cabo mediante los procesos de mediación es determinante para que el proceso de enseñanzaaprendizaje responda a la persona como ser individual, afrontando el proceso de una manera propia diferenciada de otras personas" (Martín Izard, 2014, p. 138).

\section{Tabla 5}

Nivel, tipo de tutoría virtual y descripción

\begin{tabular}{|c|c|c|}
\hline Nivel & Tipo de tutoría & Descripción \\
\hline 1 & $\begin{array}{l}\text { Tutoría virtual } \\
\text { neutra }\end{array}$ & $\begin{array}{l}\text { No hay presencia del tutor. La labor se limita a abrir y cerrar las } \\
\text { actividades y unidades didácticas. No hay respuesta hacia el estudiante. } \\
\text { Ausencia de retroalimentación. No se utilizan los medios comunicativos } \\
\text { dentro del aula virtual. }\end{array}$ \\
\hline 2 & $\begin{array}{l}\text { Tutoría virtual } \\
\text { estática }\end{array}$ & $\begin{array}{l}\text { La presencia del tutor se circunscribe a calificar las actividades de } \\
\text { los estudiantes y la retroalimentación consiste en identificar algunos } \\
\text { errores presentes en los trabajos enviados. Responde eventualmente a } \\
\text { las consultas de los estudiantes y con un lenguaje limitado. }\end{array}$ \\
\hline 3 & $\begin{array}{l}\text { Tutoría virtual } \\
\text { activa }\end{array}$ & $\begin{array}{l}\text { La acción tutorial básicamente gira en torno a la fórmula pregunta } \\
\text { - respuesta. Responde a las consultas de los estudiantes en un } \\
\text { lapso superior a las } 48 \text { horas. La retroalimentación toma como base } \\
\text { las correcciones u observaciones detectadas en el trabajo enviado } \\
\text { con algún tipo de refuerzo positivo. El estilo de comunicación suele } \\
\text { inclinarse más hacia la solución de problemas que a la empatía o } \\
\text { cercanía con el estudiante. Utiliza los medios comunicativos dentro del } \\
\text { aula virtual. }\end{array}$ \\
\hline 4 & $\begin{array}{l}\text { Tutoría virtual } \\
\text { dinámica }\end{array}$ & $\begin{array}{l}\text { Personaliza la acción tutorial por medio del apoyo constante hacia } \\
\text { el estudiante. Estimula y orienta individualmente y a nivel grupal. } \\
\text { Facilita el proceso de aprendizaje a través de los canales comunicativos } \\
\text { respectivos presentes en el aula virtual, comunicación empática. Ayuda } \\
\text { a resolver dificultades con respecto a los materiales didácticos. La } \\
\text { retroalimentación se convierte en una oportunidad de refuerzo positivo } \\
\text { sin obviar las correcciones pertinentes. Responde las consultas de los } \\
\text { estudiantes en un lapso no mayor a } 48 \text { horas. }\end{array}$ \\
\hline 5 & $\begin{array}{l}\text { Tutoría virtual } \\
\text { extrema }\end{array}$ & $\begin{array}{l}\text { Exceso de comunicación con tendencia a distraer al estudiante ante } \\
\text { tantos mensajes lo que puede desembocar en la indiferencia de este } \\
\text { ante una gran cantidad de instrucciones. Incremento sustancial de } \\
\text { material didáctico con una serie de deberes que en la práctica el } \\
\text { esfuerzo del docente se diluye y se acentúa el cansancio del estudiante. } \\
\text { Se muestra cierta inestabilidad en cuanto a las directrices acordadas } \\
\text { como producto de la desproporción informativa. Tendencia a presionar } \\
\text { constantemente al estudiante para el cumplimiento de sus deberes. }\end{array}$ \\
\hline
\end{tabular}


Ahora bien, la clasificación propuesta en la tabla 5 puede sugerir a nivel institucional una revisión profunda del proceso de formación del docente en el que se establezcan una serie de aspectos sustantivos para el aseguramiento de la calidad en el ejercicio docente dentro de los entornos virtuales. Ese proceso de formación "exige un análisis objetivo de sus necesidades y de las capacidades o competencias que debe desarrollar, brindando a partir de ello la formación especializada y adecuada a cada caso concreto" (Hernández, 2014, p. 43), por lo tanto, la clasificación expuesta en la tabla 5 muestra los matices dentro del ejercicio de la tutoría virtual.

Es importante destacar que el aprendizaje de parte del docente debe ser secuencial y debe contar con el apoyo permanente de la universidad para poder graduar y hacer los ajustes necesarios al momento de la puesta en marcha de esta acción que en definitiva puede marcar no solo la experiencia del profesor, sino que puede en algún momento determinar la prosecución de los estudios por parte de los estudiantes, por lo tanto, “las TIC pueden ofrecer una ayuda importante al profesor universitario para facilitar la necesaria renovación a las exigencias emanadas de las propuestas del EEES" (Tejedor, 2011, p. 113).

\section{CONCLUSIONES}

Una vez realizado el recorrido anteriormente descrito, y como resultado del proceso analítico e interpretativo correspondiente, es momento de desembocar en las siguientes conclusiones.

El Sistema Universitario Español ha experimentado en los últimos años una serie de modificaciones tanto en su forma como en su proporción. El contexto social, político y económico ha perfilado su radio de acción y con ello las tensiones y las transiciones producidas por una dinámica de cambio han estado presentes a lo largo del recorrido analizado hasta ahora. Los altibajos también dejan huella y solo cuando tenemos la oportunidad de mirar hacia atrás es que tenemos la ocasión de entender nuestro presente y atrevernos a proyectar un futuro entre la racionalidad y la incertidumbre.

Es oportunidad de hacer referencia ya no a la manera en que el Gobierno español se ha dirigido a la Universidad española, sino al sentido de pertenencia de los miembros de la comunidad universitaria con respecto a la institución. Más allá de coincidencias o disidencias con una determinada política pública dirigida al sector universitario, es de reflexionar sobre cómo ha evolucionado el sentimiento de apego y pertenencia a una institución que, siendo una organización, cuenta con la inobjetable misión de transmitir valores y cultura, y desde luego estimular e incentivar el saber. Por otro lado, al establecer una infranqueable zona de comodidad y seguridad personal, existe el riesgo de que los miembros que forman parte de la comunidad universitaria se aíslen dentro de la misma, ya que en definitiva y sin importar la magnitud e importancia de cualquier cambio o reforma, continuarán desempeñándose de la misma manera, con los mismos resultados, pero tal vez con menos número de estudiantes motivados.

Probablemente no todas las personas cuenten con una misma definición de lo que es la Universidad, por lo tanto, al existir inclinaciones motivadas por diversos intereses y afecciones, aunque se planteen reformas siempre existirá la tendencia a no andar por la misma senda, ya que se confrontan varias visiones sobre una misma institución que en este caso es la Universidad. Efectivamente al ser una casa de estudios de larga data, ha debido experimentar los procesos por los cuales las sociedades han tenido 
que vivir, por lo tanto, ¿Es la adaptabilidad una consecuencia o una característica de la Universidad? Al momento de descubrirse que los sistemas políticos se legitiman a través de la educación, se inició todo un proceso caracterizado por la determinación de lo que el Gobierno espera de ella en función de los intereses del Estado. Sin embargo, todas las fuerzas ideológicas no solo han influido en este particular, sino que dentro de la propia Universidad comparten un mismo espacio múltiples ideologías y conseguir para todo un acuerdo medianamente favorable es una labor casi imposible.

Es de destacar que las corrientes económicas regionales, continentales y mundiales también han tenido notoria influencia en esta adaptabilidad. Se resalta en este punto el hecho de que no significa que la Universidad al moldear su estructura y acciones deje de ser lo que es, el peligro está en que dicha adaptación se entienda a partir de que la Universidad deba abastecer de conocimiento y saber a los cuerpos decisorios que de algún modo pueden tener o tienen la capacidad de ser fuerzas de índole política y económica. En ese caso si es punto de crítica y reflexión la convicción de que el conocimiento solo pueda ser generado en cuanto aporte valor agregado al mercado. La referencia de la Universidad es la sociedad y no el mercado.

Una de las maneras de mantener viva la institución universitaria es la generación del saber y su posterior difusión. La investigación en este caso cobra una importancia singular, ya que esta acción es la que acompaña indivisiblemente la misión del educador universitario, sin embargo, ¿ todo investigador cuenta con vocación y formación docente? Tal y como se ha reflexionado en este trabajo, la cantidad o calidad de la investigación no es sinónimo de formación para el ejercicio docente. Aunado a ello, el estímulo y apoyo a la vocación del educador universitario es un aspecto que bajo ninguna circunstancia ha de ser descuidado.

En este particular el uso y empleo de las tecnologías de la información y la comunicación se constituye en una herramienta y un medio, no en un fin. Por lo tanto, aunque contemos con capacitación tecnológica, si no se tiene convicción de la importancia de la función docente ninguna reforma universitaria será suficiente para la mejora de la Universidad. En este caso somos los propios académicos los que debemos reforzar esta idea sin esperar que otros lo hagan antes que nosotros sin la seguridad de saber que aquellos que deciden, cuenten con un claro sentido universitario, académico, docente e investigador.

De tal manera, uno de los retos a los que se enfrenta el mundo docente desde el reconocimiento de la nueva concepción de su acción, es la formación del profesorado universitario (Rodríguez-Moneo, 2011), ya que constituye un aspecto de vital importancia de cara al desarrollo de la reforma del sistema universitario europeo y su efecto inminente en el sector universitario español. De tal manera, en el EEES, Rodríguez-Moneo (2011) identifica dos aspectos relevantes en función de dicha actualización docente. Uno de ellos es el nuevo enfoque que se propone en la formación universitaria que se entiende a partir del énfasis en el proceso de aprendizaje. El otro aspecto tiene que ver con los cambios metodológicos que se presentan en el EEES, principalmente a partir de la formación por competencias.

A propósito de este punto, a nivel global no se ha identificado una instrucción que explícitamente sugiera o indique sobre la formación del profesorado universitario europeo en cuanto a la actualización de la didáctica respectiva, aunque se propone que las universidades en su autonomía académica 
puedan diseñar estrategias y acciones vinculadas con estos aspectos. Sin embargo, esta imprecisión puede generar que la necesidad de una formación adecuada en cuanto a los requerimientos didácticos que exige el nuevo escenario educativo en Europa y desde luego en España, sea considerada poco llamativa no solo por los cuerpos decisorios, sino por los propios profesores universitarios. Además de lo anterior, "tradicionalmente, a los profesores universitarios no se les exige una formación didáctica o instruccional y sólo se forman como expertos en las disciplinas que imparten”. (Rodríguez-Moneo, 2011, p. 21).

Es tal vez un ejercicio arriesgado responder a la pregunta ¿Hacia dónde va la Universidad española?, o más bien, ¿Hacia dónde quieren que vaya la Universidad española? Algunas constataciones identificadas a lo largo de esta investigación nos pueden ayudar a dibujar un posible panorama. En primer lugar, y sin ánimos demagógicos, la reducción de las asignaciones presupuestarias han afectado todas las instancias del quehacer universitario desde la reposición de los cargos, la contratación de nuevos profesores, el crecimiento de la investigación, el afianzamiento de la Universidad como campus de excelencia internacional, la reducción de las ayudas económicas a los estudiantes, las estancias pre y posdoctorales y el aumento del precio de las matrículas aleja cada vez más que se considere al estudiante como epicentro de la Universidad tal y como lo establece el proceso de Bolonia y logrando con esto que el sector privado de la economía ofrezca posibilidades económicas al sector universitario favoreciendo el crecimiento de las disciplinas o campos de estudios que dicho sector necesita.

Junto a este panorama, el peso de la tradición que reposa sobre algunos universitarios va acompañado de una posición indiferente ante lo que reamente necesita la Universidad, ocasionando así que se forme al estudiantado de la misma manera sin ninguna señal de cambio y por lo tanto el mismo patrón se repite al momento de que esos estudiantes algún día tengan la oportunidad de enseñar. Sin dejar de mencionar la confusión de algunos dirigentes universitarios al momento de defender sus espacios de acción confundiendo lo multidisciplinario con un plan de puertas abiertas a todas las disciplinas para que se asegure un número de estudiantes en un determinado programa, pero que en la práctica se traduce en que un mismo fenómeno objeto de estudio sea analizado desde una sola perspectiva, desconociendo el valor de otras metodologías que acompañan a los diversos campos de estudio.

Es de destacar que muchas universidades han impulsado planes de formación dirigidos al profesorado con el objeto de formarles no solo en el uso y empleo didáctico de las nuevas tecnologías, sino que también se promuevan iniciativas compatibles directamente con el reconocimiento de la nueva propuesta didáctica que deriva de la ejecución de Bolonia. Además de ello es también de señalar que esta formación tiene carácter voluntario y los índices de participación, aunque se mantienen, tienden a la baja. Con respecto a los profesores de nuevo ingreso se reconoce una participación superior con relación a los profesores que ya cuentan con varios años dentro de la Universidad. El profesorado de nuevo ingreso está recibiendo, por consiguiente, la formación didáctica propia del EEES, es más, algunas universidades hacen concursos internos para la promoción de los proyectos de innovación docente en función de Bolonia.

Desde luego, son muchas las aristas por considerar al momento de tratar el tema de la formación docente, además de los factores intrínsecos, extrínsecos y personales que influyen en la persona del 
profesor universitario, no obstante, uno de los objetivos de esta investigación es aproximar al lector a una serie de aspectos conceptuales, actitudinales y analíticos en relación con el reconocimiento de la relevancia e implicaciones de la tutoría virtual. La tutoría virtual se identifica como una de las acciones más determinantes dentro de la educación en línea o virtual. En este sentido el factor motivacional y el compromiso con la labor formadora por parte del docente pueden incidir en el interés y estímulo del estudiante que viene generalmente de una educación presencial. Así mismo, es un reto el lograr que el estudiante universitario sea consciente que es el administrador de su propio proceso de aprendizaje y es allí en donde el tutor virtual hace presencia, pero ya no como transmisor de conocimientos sino como facilitador de procesos del futuro profesional universitario. Sin embargo, y aunque se ofrezca la preparación didáctica necesaria, sin vocación docente, ningún esfuerzo será suficiente.

Es un hecho el que la tutoría virtual y el despliegue de su acción incluya aspectos tales como: el acercamiento al saber de parte del estudiante, la guía y motivación con relación al proceso de aprendizaje con el objeto de asimilar aquellas competencias establecidas en el currículum, estimular y fomentar la actividad investigadora y la búsqueda de información y la equilibrada y orientada elección de planes e itinerarios de formación. Estas consideraciones nos permiten afirmar que el rol del profesor universitario como tutor virtual ofrece una serie de posibilidades y retos de corte procedimental y actitudinal ya que al aceptar que la acción tutorial es una característica propia de la función docente, en el contexto virtual, el acompañamiento y su intensidad va a estar mediado por aquella disposición y preparación didáctica que facilite el proceso orientador desde una visión sistémica y orientadora, a la par del acercamiento del estudiante a la disciplina o campo de estudio que esté cursando. El nivel y tipo de tutoría virtual aquí expuesto va a servir para identificar el grado de realización de esta acción tutorial, con el propósito de identificar lógicamente los retos y fortalezas, de manera que por ejemplo al diseñar planes formativos dirigidos al profesorado universitario o al hacer seguimiento y acompañamiento a los profesores, estos también puedan percibir el efecto positivo de la orientación y acompañamiento efectivo para que dicho efecto pueda revertirse a los estudiantes.

Es el momento en que debemos decidir hacia dónde va la Universidad española. Desde nuestros espacios de trabajo podemos identificar dos opciones: o permitir que la Universidad se convierta en una dispensadora de títulos, o permitir que la evolución de la Universidad no modifique su esencia ni su razón de ser. Hay que tocar fondo, llegar a la fibra de cada miembro de la comunidad universitaria. Tanto la euforia como el desánimo colectivo son altamente contagiosos y alcanzar un equilibrio entre la racionalidad y la incertidumbre es un reto permanente. Es inconcebible la indiferencia ante una metamorfosis institucional que pueda significar la variación del ser de la Universidad. El ímpetu y la convicción no pueden venir desde fuera, sino de cada uno de los académicos. En estos tiempos la apatía no se puede considerar como opción y por lo tanto la solidez de los cimientos de la Universidad no pueden ser entendidos ni defendidos por alguien que no la sienta propia, por lo tanto, la Universidad al ser una especie de órgano vivo en constante evolución va creciendo conforme sus integrantes lo hagan y es desde ese punto en que los universitarios podremos sincronizar nuestros pasos con los de una institución única y diversa, particular y universal. 


\section{REFERENCIAS}

Angoitia, M. \& Rahona, M. (2007). Evolución de la educación universitaria en España: diferentes perspectivas y principales tendencias (19912005). Revista de Educación, 344, 245-264. Recuperado de https://goo.gl/WbQQTd

Borges, F. (2005). La frustración del estudiante en línea. Causas y acciones preventivas. Digithum, 7. Recuperado de https://goo.gl/t6AEXU

Cabrera, L. (2015). Efectos del proceso de Bolonia en la reducción del abandono de estudios universitarios: datos para la reflexión y propuestas de mejora. Revista Fuentes, 16, 39-62. Recuperado de https://goo.gl/uQWXov

Dávila, M. (2008). Tendencias internacionales de la Educación Superior. Documento de Trabajo N²19, Universidad de Belgrano. Recuperado de https://goo.gl/YWUCok

Del Pozo Andrés, M. (2008). El proceso de Bolonia en las aulas universitarias: una perspectiva europea. Cuestiones Pedagógicas, 19 , 55-73. Recuperado de https://goo.gl/chcSr3

Dopico Rodríguez, E. (2013). Tutoría universitaria: propuestas didácticas de competencia tutorial. REDU. Revista de Docencia Universitaria, 11(2), 195-220. Recuperado de https://goo.gl/ybjbsi

Fernández Enguita, M. (1999). La transformación de la Universidad española. Revista Interuniversitaria de Formación del Profesorado, 34,31 -37. Recuperado de https://goo.gl/rnWVju

Giner, Y., Muriel, M. \& Toledano, F. (2013). De la tutoría presencial a la virtual: la evolución del proceso de tutorización. REDU. Revista de Docencia Universitaria, 11(2), 89-106. Recuperado de https://goo.gl/xqFpx9

Hernández, A. (2014). La formación del profesorado en el proceso de integración de las TIC en el currículum: nuevos roles, competencias y espacios de formación. En A. García-Valcárcel (Coord.), Investigación y tecnología de la información y comunicación al servicio de la innovación educativa (pp. 33-56). Salamanca: Ediciones Universidad de Salamanca.

Hernández, A. \& Martín, J. (2011). El punto de vista del profesorado sobre la integración de las TIC en la docencia. En A. García-Valcárcel (Coord.), Integración de las TIC en la docencia universitaria (pp. 167-182). Salamanca: Netbiblo.

Kindelán, M. P. (2013). Una perspectiva sobre el binomio enseñanza-investigación en la universidad del s. XXI. Revista Complutense de Educación, 24(1), 27-45. Recuperado de https://goo.gl/qY6nWw

Llinàs-Audet, X., Girotto, M. \& Salé, F. (2011). La dirección estratégica universitaria y la eficacia de las herramientas de gestión: el caso de las universidades españolas. Revista de Educación, 355, 33-54. Recuperado de https://goo.gl/szUkQR

López, M., Pérez-García, M. \& Rodríguez, M. (2015). Concepciones del profesorado universitario sobre la formación en el marco del espacio europeo de educación superior. Revista de Investigación Educativa, 33(1), 179-194. doi: http://dx.doi.org/10.6018/rie.33.1.189811

Martín Izard, J. F. (2014). Tutoría en espacios virtuales de aprendizaje y procesos de mediación didáctica. En A. García-Valcárcel (Coord.), Investigación y tecnología de la información y comunicación al servicio de la innovación educativa (pp. 113-140). Salamanca: Ediciones Universidad de Salamanca.

Martínez Carrazo, P. (2006). El método de estudio de caso: estrategia metodológica de la investigación científica. Pensamiento \& Gestión, 20, 165-193. Recuperado de https://goo.gl/9ozdaW

Martínez, P., Pérez, J. \& Martínez Juárez, M. (2014). Una (re) visión de la tutoría universitaria en los estudios de Grado. REDU. Revista de Docencia Universitaria, 12(1), 269-305. Recuperado de https://goo.gl/wti8Po

Quintero, A. (2014). Innovación educativa e integración curricular de las TIC. En A. García-Valcárcel (Coord.), Investigación y tecnología de la información y comunicación al servicio de la innovación educativa (pp. 9-32). Salamanca: Ediciones Universidad de Salamanca.

Rodríguez, N. (2014). El tutor frente a la educación a distancia: concepciones, funciones y estrategias tutoriales. Revista de educación, cooperación y bienestar social, 3, 49-69. Recuperado de https://goo.gl/WUx2f8

Rodríguez-Moneo, M. (2011). El proceso de enseñanza y aprendizaje de competencias. En F. Labrador \& R. Santero (Eds.), Evaluación global de los resultados de aprendizaje en las titulaciones dentro del Espacio Europeo de Educación Superior (pp 19- 43) Madrid: Dykinson.

Roselló, G., Olivares, I. \& Pujolras, O. (2009). Informe sobre el Sistema de Educación Superior y los Mecanismos de Aseguramiento de la Calidad en España. Barcelona: Agencia para la Calidad de la Universitat de Barcelona.

Ruiz-Gallardo, J. R. \& Castaño, S. (2008). La Universidad española ante el reto del EEES. Docencia e Investigación: Revista de la Escuela Universitaria de Magisterio de Toledo, 18, 253-270. Recuperado de https://goo.gl/LmnRTS

Sandín, M. (2003). Investigación cualitativa en educación, fundamentos y tradiciones. Madrid: McGraw-Hill.

Sanjurjo, V. (2012). El proceso de Bolonia: mito y realidad. Revista Jurídica de Investigación e Innovación Educativa, 5, 121-146. Recuperado de https://goo.gl/aES7jv 
Santoveña, S. (febrero, 2010). Innovación didáctica en el marco del Espacio Europeo de Educación Superior. Trabajo presentado en el II Congrés Internacional de Didáctiques, Girona, Cataluña. Recuperado de https://goo.gl/P6DHFs

Tejedor, F. (2011). Estrategia de aprendizaje y uso de las TIC. En A. García-Valcárcel (Coord.), Integración de las TIC en la docencia universitaria (pp. 105-118). Salamanca: Netbiblo.

Tejedor, F. (2014). Investigación educativa: su desarrollo en el ámbito de la tecnología educativa. En A. García-Valcárcel (Coord.), Investigación y tecnología de la información y comunicación al servicio de la innovación educativa (pp. 176-208). Salamanca: Ediciones Universidad de Salamanca.

Toledo Lara, G. (2015). La universidad española y el proceso de Bolonia: consideraciones para su análisis. Actualidades Investigativas en Educación, 15(2), 1-23. doi: http://dx.doi.org/10.15517/aie.v15i2.18968

Toledo Lara, G. (2017). La investigación cualitativa y el estudio de casos: una revisión teórica para su revisión. Revista Dilemas Contemporáneos: Educación, Política y Valores, 4(2). Recuperado de https://goo.gl/F04ps7

(c) Los autores. Este artículo es publicado por la Revista Digital de Investigación en Docencia Universitaria del Área de Institutional Research and Effectiveness de la Dirección de Aseguramiento de la Calidad, Universidad Peruana de Ciencias Aplicadas. Este es un artículo de acceso abierto, distribuido bajo los términos de la LicenciaCreativeCommons Atribución-Compartirlgual 4.0 Internacional.I http://creativecommons.org/licenses/bysa/4.0/I, que permite el uso no comercial, distribución y reproducción en cualquier medio, siempre que la obra original sea debidamente citada. 